\title{
Substituição do farelo de soja pela torta de babaçu em rações para frangos de corte dos 22 aos 42 dias de idade ${ }^{1}$
}

\author{
Replacement of soybean meal with babassu meal in rations for \\ broilers from 22 to 42 days old
}

\author{
Mônica Calixto da Silva ${ }^{2}$; Jefferson Costa de Siqueira ${ }^{3 *}$; \\ Roberta Gomes Marçal Vieira Vaz ${ }^{4}$; Kênia Ferreira Rodrigues \\ Ana Cláudia Gomes Rodrigues Neiva ${ }^{4}$; Gerson Fausto da Silva ${ }^{4}$; \\ Flávia Luzia Rodrigues Fonseca²; Joana Patrícia Lira de Sousa²; \\ Carla Fonseca Alves ${ }^{5}$; Luciana Pereira Nabute Cunha ${ }^{6}$
}

\section{Resumo}

O objetivo deste trabalho foi avaliar a viabilidade técnica e econômica da substituição parcial do farelo de soja pela torta de babaçu em rações para frangos de corte dos 22 aos 42 dias de idade. Foram utilizados 80 frangos Cobb $500^{\circledR}$, distribuídos em delineamento inteiramente casualizado, com quatro tratamentos $(0,10,20$ e $30 \%$ de substituição do farelo de soja pela torta de babaçu) e cinco repetições de quatro aves. Foram avaliados o desempenho (ganho de peso, consumo de ração e conversão alimentar), rendimentos de carcaça e cortes, biometria dos órgãos, custo com alimentação por kg de frango produzido e margem bruta. Para verificar as relações de custo da substituição do farelo de soja pela torta de babaçu, foram estabelecidas inequações. Os níveis de substituição do farelo de soja pela torta de babaçu não influenciaram $(\mathrm{P}>0,05)$ nenhuma das características de desempenho, o que evidenciou a viabilidade técnica da substituição de até $30 \%$ do alimento. Da mesma forma, não houve efeito sobre os rendimentos de carcaça, cortes e biometria dos órgãos. O menor custo com alimentação por $\mathrm{kg}$ de frango produzido e a maior margem bruta foram obtidos com a utilização da ração contendo $0 \%$ de torta de babaçu. $\mathrm{O}$ aumento do nível de substituição do farelo de soja pela torta de babaçu, nas rações para frangos de corte dos 22 aos 42 dias de idade mostrou-se economicamente inviável, porém, as inequações elaboradas podem ser de grande utilidade em situações práticas.

Palavras-chave: Alimento alternativo, coprodutos do babaçu, custo da alimentação, desempenho produtivo

\footnotetext{
Abstract

The objective this work was to evaluate the technical and economic viability of the substitution level of soybean meal by babassu meal in rations to broiler from 22 to 42 days old. It was used 80 male broiler

${ }^{1}$ Parte da Dissertação de Mestrado do primeiro autor, Universidade Federal do Tocantins, UFT, Araguaína, TO.

2 Discentes do Curso de Doutorado, Programa de Pós-Graduação em Ciência Animal Tropical, Universidade Federal do Tocantins, UFT, Araguaína, TO. E-mail: monicalixto@hotmail.com; flrf@outlook.com;.jp-zoot@hotmail.com

3 Prof. Dr., Universidade Federal do Maranhão, UFMA, Centro de Ciências Agrárias e Ambientais, Chapadinha, MA, Bolsista FAPEMA. E-mail: jcsiqueira@ufma.br

${ }^{4}$ Profs. Drs., Dept ${ }^{\circ}$ de Zootecnia, Escola de Medicina Veterinária e Zootecnia, UFT, Araguaína, TO. E-mail: betagmvvaz@yahoo. com.br; rodrigueskf@mail.uft.edu.br; aclaudianeiva@gmail.com; gerson@mail.uft.edu.br

5 Discente do Curso de Mestrado, Programa de Pós-Graduação em Ciência Animal Tropical, UFT, Araguaína, TO. E-mail: carlafazoo@hotmail.com

${ }^{6}$ Discente do Curso de Zootecnia, UFT, Araguaína, TO. E-mail: luciananabute@hotmail.com
}

* Autor para correspondência 
chicks at one day of age, distributed into complete random designs with four treatments $(0,10,20$ e $30 \%$ substituition of soybean meal by babassu meal) and five repetitions of four broilers each. Were evaluated the performance (weight dain, feed intake and feed conversion), carcass and cuts, organ biometry, feed cost per kilogram body weight and gross margin. To verify the relationship of cost of replacing soybean meal with pie babassu, inequalities were established. The substitution level of soybean meal by babassu meal had no influence $(\mathrm{P}>0,05)$ any of the performance characteristics, wich showed the technical viability of substituting up to $30 \%$. Similarly, there was no affect on carcass yield, cuts weight and organ biometry. The cust less with feed per kg for chicken produced and the higher gross margin were obtained from chickens fed diets with $0 \%$ babassu meal. The increased of substitution level soybean meal by babassu meal in ration for broilers from 22 to 42 days old proved unviable economically, however, the inequalities produced can be useful in practical situations.

Key words: Alternative food, coproducts of babassu, feed cost, productive performance

\section{Introdução}

A sazonalidade de matéria prima para confecção de rações tem despertado o interesse dos nutricionistas por fontes alternativas de nutrientes capazes de substituir o milho e o farelo de soja na alimentação das aves, sem comprometer o desempenho (BASTOS et al., 2007; RAMOS et al., 2006; BRUNELLI et al., 2006; NASCIMENTO et al., 2005). Dentre eles, destacam-se os coprodutos do coco do babaçu, pois o processamento industrial do coco do babaçu para extração do óleo da amêndoa gera a torta (resultante do processo de prensagem) e o farelo do babaçu (oriundo do processo em que se utiliza solvente químico).

Estudos recentes foram desenvolvidos com a utilização desses coprodutos e demonstraram potencialidades para serem utilizados na alimentação das aves, especificamente, pela possibilidade de reduzir a utilização do milho e do farelo de soja, principalmente na entressafra, período em que geralmente esses ingredientes encontram-se em escassez e com preços mais elevados.

Carneiro et al. (2009) não verificaram efeitos de níveis crescentes de inclusão do farelo de babaçu $(3,6,9$ e $12 \%)$ sobre o consumo de ração, ganho de peso, conversão alimentar e rendimento de carcaça de frangos de corte na fase de 21 a 42 dias de idade, demonstrando a viabilidade técnica de utilização desta fonte de nutrientes.

Em estudo similar, Santos Neta et al. (2011) avaliaram níveis crescentes de inclusão da torta de babaçu $(0,4,8$ e $12 \%)$ em rações de frangos de corte de um a 21 dias e não verificaram efeitos dos tratamentos sobre nenhuma das características de desempenho, o que evidenciou a possibilidade de inclusão de até $12 \%$ deste alimento nas rações.

Os diferentes estudos mostraram a eficácia da utilização da torta de babaçu em rações para frangos de corte, em diferentes fases de produção. Entretanto, deve-se enfatizar que a utilização desse alimento em escala comercial, depende de análises de economicidade para avaliar qual nível de inclusão do alimento proporciona menor custo com alimentação e máxima lucratividade. Diante do exposto, objetivou-se, avaliar a viabilidade técnica e econômica da substituição parcial do farelo de soja pela torta de babaçu em rações para frangos de corte dos 22 aos 42 dias de idade.

\section{Material e Métodos}

O experimento foi conduzido no Setor de Avicultura da Escola de Medicina Veterinária e Zootecnia da Universidade Federal do Tocantins, localizado em Araguaína - TO, no período de 17 de fevereiro a 08 de março de 2012. Trabalho aprovado pela Comissão de Ética no Uso de Animais da Universidade Federal do Tocantins (CEUA-UFT), processo $n^{\circ} 23101.001308 / 2013-62$.

Foram utilizados 80 frangos de corte, da linhagem Cobb $500^{\circledR}$, que foram criados em galpão 
experimental, coberto com de palha de babaçu e piso de concreto, em gaiolas de $0,5 \times 0,5 \times 0,5$ $\mathrm{m}$, com comedouros e bebedouros tipo calha. $\mathrm{O}$ abastecimento dos comedouros, a limpeza e o reabastecimento dos bebedouros foram realizados duas vezes por dia, visando garantir o livre acesso à água e as rações durante todo o período experimental.

As condições ambientais, no interior das instalações durante o período experimental, foram monitoradas e registradas diariamente utilizandose os termômetros de bulbo seco, bulbo úmido e de máxima/mínima; colocados a meia altura das gaiolas, possibilitando os cálculos das temperaturas média, máxima, mínima e da umidade relativa do ar.
As aves foram alimentadas com ração formulada para atender as exigências nutricionais de acordo com Rostagno et al. (2011) até o $21^{\circ}$ dia de vida. No $22^{\circ}$ dia, as aves com peso médio de $890 \pm 69,17 \mathrm{~g}$, foram distribuídas em delineamento experimental inteiramente casualizado (DIC), com quatro tratamentos $(0,10,20$ e $30 \%$ de substituição do farelo de soja pela torta de babaçu) e cinco repetições de quatro aves por unidade experimental.

As rações experimentais foram calculadas considerando a composição química dos ingredientes (Tabela 1) e as exigências nutricionais das aves de acordo com as recomendações de Rostagno et al. (2011) (Tabela 2).

Tabela 1. Composição dos principais ingredientes utilizados na formulação das rações experimentais (com base na matéria natural).

\begin{tabular}{lccc}
\hline \multicolumn{1}{c}{ Nutriente } & Milho & Farelo de Soja & Torta de babaçu \\
\hline Energia bruta (kcal/kg) & $3983^{1}$ & $4340^{1}$ & $4556^{1}$ \\
Energia metabolizável (kcal/kg) & $3381^{3}$ & $2254^{3}$ & $2268^{2}$ \\
Proteína bruta (\%) & $9,04^{1}$ & $44,06^{1}$ & $21,26^{1}$ \\
Cálcio (\%) & $0,03^{3}$ & $0,24^{3}$ & $0,12^{3}$ \\
Fósforo disponível (\%) & $0,06^{3}$ & $0,22^{3}$ & $0,41^{3}$ \\
Lisina digestível (\%) & $0,20^{3}$ & $2,57^{3}$ & $0,38^{3}$ \\
Metionina+cistina digestível (\%) & $0,32^{3}$ & $1,13^{3}$ & $0,31^{3}$ \\
Metionina digestível (\%) & $0,16^{3}$ & $0,55^{3}$ & $0,37^{3}$ \\
Treonina digestível (\%) & $0,29^{3}$ & $1,57^{3}$ & $0,35^{3}$ \\
Sódio (\%) & $0,02^{3}$ & $0,02^{3}$ & $0,06^{3}$ \\
Potássio (\%) & $0,29^{3}$ & $1,83^{3}$ & $0,48^{3}$ \\
Cloro (\%) & $0,06^{3}$ & $0,05^{3}$ & ----- \\
Fibra bruta (\%) & $1,97^{1}$ & $9,67^{1}$ & $29,21^{1}$ \\
Fibra em detergente neutro (\%) & $13,85^{1}$ & $21,73^{1}$ & $58,63^{1}$ \\
Fibra em detergente ácido (\%) & $3,16^{1}$ & $6,74^{1}$ & $30,64^{1}$ \\
\hline
\end{tabular}

${ }^{1}$ Análises realizadas no Laboratório de Nutrição Animal da Escola de Medicina Veterinária e Zootecnia da Universidade Federal do Tocantins.

${ }^{2}$ Santos Neta (2010).

${ }^{3}$ Rostagno et al. (2011).

Fonte: Elaboração dos autores.

As variáveis avaliadas foram consumo de ração (CR), ganho de peso (GP), conversão alimentar (CA), peso final (PF), biometria dos órgãos (coração, fígado, moela e intestino delgado), rendimento de carcaça $(\mathrm{RC})$ e rendimentos de cortes nobres (coxa, sobrecoxa e peito). 
As aves foram pesadas no início e no final do período experimental para determinação do ganho de peso. $\mathrm{O}$ consumo de ração foi calculado considerando a quantidade de ração fornecida e as sobras nos comedouros. A conversão alimentar foi calculada pela razão entre o consumo de ração e o ganho de peso das aves.

Aos 42 dias de idade, duas aves de cada parcela, com peso corporal próximo ao da média da parcela $( \pm 5 \%)$, foram submetidas a jejum alimentar de 12 horas e abatidas por deslocamento cervical. Em seguida, foram submetidas aos procedimentos de sangria, escaldagem, depenagem e evisceração, para avaliação dos pesos relativos (\%) das carcaças inteiras (com pés, pescoço e cabeça) e dos cortes nobres (coxa, sobrecoxa e peito).

Tabela 2. Composição das rações experimentais contendo níveis crescentes de substituição do farelo de soja pela torta de babaçu para frangos de corte de 22 a 42 dias de idade.

\begin{tabular}{|c|c|c|c|c|}
\hline \multirow{2}{*}{ Ingredientes } & \multicolumn{4}{|c|}{ Níveis de substituição do farelo de soja pela torta de babaçu (\%) } \\
\hline & 0 & 10 & 20 & 30 \\
\hline Milho & 64,689 & 64,689 & 64,689 & 64,689 \\
\hline Farelo de Soja (45\%) & 27,971 & 25,174 & 22,377 & 19,580 \\
\hline Torta de Babaçu & 0,000 & 2,797 & 5,594 & 8,391 \\
\hline Fosfato bicálcico & 1,162 & 1,163 & 1,165 & 1,166 \\
\hline Óleo de soja & 2,473 & 2,782 & 3,092 & 3,401 \\
\hline Calcário & 0,749 & 0,767 & 0,784 & 0,802 \\
\hline Sal comum & 0,402 & 0,403 & 0,403 & 0,404 \\
\hline DL-Metionina & 0,242 & 0,266 & 0,289 & 0,312 \\
\hline L-Lisina $\mathrm{HCl}$ & 0,261 & 0,343 & 0,423 & 0,505 \\
\hline L-Treonina & 0,053 & 0,088 & 0,123 & 0,158 \\
\hline Suplemento mineral e vitamínico ${ }^{1}$ & 0,500 & 0,500 & 0,500 & 0,500 \\
\hline Areia lavada (inerte) & 1,500 & 1,025 & 0,565 & 0,088 \\
\hline Total & 100,00 & 100,00 & 100,00 & 100,00 \\
\hline \multicolumn{5}{|c|}{ Composição nutricional calculada } \\
\hline EM (kcal/kg) & 3100 & 3100 & 3100 & 3100 \\
\hline Proteína bruta (\%) & 18,75 & 18,16 & 17,56 & 16,97 \\
\hline Cálcio (\%) & 0,685 & 0,685 & 0,685 & 0,685 \\
\hline Fósforo Disponível (\%) & 0,320 & 0,320 & 0,320 & 0,320 \\
\hline Lisina Digestível (\%) & 1,044 & 1,044 & 1,044 & 1,044 \\
\hline Metionina + cistina Digestível (\%) & 0,762 & 0,762 & 0,762 & 0,762 \\
\hline Metionina Digestível (\%) & 0,506 & 0,523 & 0,538 & 0,554 \\
\hline Treonina Digestível (\%) & 0,678 & 0,678 & 0,678 & 0,678 \\
\hline Fibra bruta $(\%)$ & 3,979 & 4,526 & 5,072 & 5,619 \\
\hline Fibra em detergente neutro (\%) & 15,038 & 16,070 & 17,102 & 18,134 \\
\hline Fibra em detergente ácido (\%) & 3,929 & 4,598 & 5,266 & 5,935 \\
\hline Sódio $(\%)$ & 0,198 & 0,198 & 0,198 & 0,198 \\
\hline Cloro $(\%)$ & 0,290 & 0,290 & 0,290 & 0,290 \\
\hline Potássio (\%) & 0,699 & 0,662 & 0,624 & 0,586 \\
\hline
\end{tabular}

${ }^{1}$ Ácido Fólico 120,00 mg, Cobalto 179,00 mg, Cobre 2.688,00 mg, Colina 108,00 g, Ferro 11,00 g, Iodo 537,00 mg, Lincomicina 800,00 mg, Manganês 31,00 g, Matéria mineral 350,00 g, Niacina 6.000,00 mg, Pantotenato de Cálcio $1.920,00 \mathrm{mg}$, Salinomicina 12,00 g, Selênio 54,00 mg, Umidade $80.00 \mathrm{~g}$, Vitamina A 1.500.000,00 UI, Vitamina B1 300,00 mg, Vitamina B12 2.800,00 mg, Vitamina B2 960,00 mg, Vitamina B6 450,00 mg, Vitamina D3 300.000,00 UI, Vitamina E 3.000,00 UI, Vitamina H 20,00 mg, Vitamina K 480,00 mg, Zinco 22,00 g.

Fonte: Elaboração dos autores. 
As vísceras comestíveis (moela, coração e fígado) e o intestino delgado, foram coletados durante a evisceração, limpos, secos em papel toalha e pesados separadamente em balança de precisão. Da moela, foi removida toda a gordura aderida, seu conteúdo e a membrana coilina. Além do peso, foi medido o comprimento do intestino delgado do início do duodeno até a junção íleocecal. O peso relativo da carcaça depenada e eviscerada foi calculado em relação ao peso em jejum. Os pesos relativos dos cortes, das vísceras comestíveis e do intestino delgado foram obtidos em relação à carcaça depenada e eviscerada.

Os dados das variáveis avaliadas foram submetidos aos testes de Normalidade (Cramer Von Mises) e Homocedasticidade (Levene). Satisfeitas essas pressuposições, as variáveis foram submetidas à análise de variância segundo o modelo estatístico: 4,5 .

$$
Y_{i j}=\mu+N S_{i}+e_{i j ;} \operatorname{com~i}=1,2,3,4 ; j=1,2,3,
$$

Em que $\mathrm{Y}_{\mathrm{ij}}=$ valor observado para a variável de interesse nas aves da j-ésima repetição recebendo o i-ésimo nível de substituição do farelo de soja pela torta de babaçu; $\mu=$ efeito da média geral; $\mathrm{NS}_{\mathrm{i}}=$ efeito do i-ésimo nível de substituição do farelo de soja pela torta de babaçu, $\mathrm{e}_{\mathrm{ij}}=$ erro experimental. As análises estatísticas foram realizadas com o auxílio do Software SAS 9.0 (2002).

Os custos com a alimentação foram determinados considerando-se, que os tratamentos foram aplicados em sistemas de produção que demandavam os mesmos insumos, diferindo apenas quanto as rações fornecidas, utilizando-se então, para quantificar o diferencial de custos entre um tratamento e outro, somente o cálculo das despesas na alimentação das aves (PEREIRA et al., 2003).

Para comparar a eficiência econômica entre as rações experimentais determinou-se o custo com alimentação por $\mathrm{kg}$ de frango produzido como segue:

$$
\mathrm{CF}_{\mathrm{i}}=\left(\mathrm{QR}_{\mathrm{i}} \times \mathrm{CR}_{\mathrm{i}}\right) / \mathrm{GP}_{\mathrm{i}} \text {; } \operatorname{com} \mathrm{i}=1,2,3,4 .
$$

Em que $\mathrm{CF}_{\mathrm{i}}=$ custo da alimentação por $\mathrm{kg}$ de frango produzido com a utilização do i-ésimo nível de substituição do farelo de soja pela torta de babaçu $(\mathrm{R} \$ / \mathrm{kg}), \mathrm{QR}_{\mathrm{i}}=$ quantidade de ração consumida no i-ésimo nível de substituição do farelo de soja pela torta de babaçu $(\mathrm{kg}), \mathrm{CR}_{\mathrm{i}}=$ custo da ração contendo o i-ésimo nível de substituição do farelo de soja pela torta de babaçu $(\mathrm{R} \$ / \mathrm{kg})$ e $\mathrm{GP}_{\mathrm{i}}=$ é o ganho de peso das aves que receberam o i-ésimo nível de substituição do farelo de soja pela torta de babaçu (kg).

A margem bruta em relação ao custo da alimentação por $\mathrm{kg}$ de frango para cada nível de substituição do farelo de soja pela torta de babaçu nas rações foi calculada pela expressão: $\mathrm{MB}_{\mathrm{i}}=\mathrm{PVF}$ - $\mathrm{CF}_{\mathrm{i}}$; em que $\mathrm{MB}_{\mathrm{i}}=$ margem bruta em relação ao custo da alimentação por $\mathrm{kg}$ de frango obtida com a utilização do i-ésimo nível de substituição do farelo de soja pela torta de babaçu (R\$); PVF = preço de venda do frango vivo $(\mathrm{R} \$ / \mathrm{kg})$ e $\mathrm{CF}_{\mathrm{i}}=$ custo por $\mathrm{kg}$ de frango produzido com a utilização do i-ésimo nível de substituição do farelo de soja pela torta de babaçu ( $\mathrm{R} \$ / \mathrm{kg})$.

Para verificar as relações de custo da substituição do farelo de soja pela torta de babaçu foram estabelecidas inequações baseadas nos custos decorrentes da redução da quantidade de farelo de soja e do aumento da quantidade de torta de babaçu nas rações, como segue:

$\left(\right.$ RFS $\left._{\mathrm{i}} \times \mathrm{PFS}\right)>\left[\left(\mathrm{ATB}_{\mathrm{i}} \times \mathrm{PTB}\right)+\left(\mathrm{AOS}_{\mathrm{i}} \times \mathrm{POS}\right)+\right.$ $\left(\mathrm{AD}-\mathrm{LM}_{\mathrm{i}} \times \mathrm{PDL}-\mathrm{Met}\right)+\left(\mathrm{AL}-\mathrm{Lis}_{\mathrm{i}} \mathrm{x}\right.$ PL-Lis $)+(\mathrm{AL}-$ Treo $_{\mathrm{i}} \times$ PL-Treo)]; com $\mathrm{i}=1,2,3,4$.

Em que $\mathrm{RFS}_{\mathrm{i}}=$ coeficiente que representa a redução da quantidade de farelo de soja na ração no i-ésimo nível de sua substituição pela torta de babaçu, obtido da pela expressão: (Nível de farelo de soja (\%) na ração controle ( $0 \%$ de substituição) Nível de farelo de soja (\%) nas rações com o i-ésimo nível de substituição (10, 20 e 30\%)) / 100 ; ATB $_{i}$; $\operatorname{AOS}_{\mathrm{i}} ;{\mathrm{AD}-L M_{\mathrm{i}}}_{\mathrm{i}} \mathrm{AL}^{\mathrm{A}-\mathrm{Lis}_{\mathrm{i}} \text { e AL-Treo }}{ }_{\mathrm{i}}=$ coeficientes que representam o aumento da quantidade de torta de babaçu, óleo de soja, DL-metionina, L-lisina 
$\mathrm{HCl}$ e L-treonina, respectivamente, no i-ésimo nível de substituição do farelo de soja nas rações, obtidos pela expressão: Nível do ingrediente (\%) nas rações com o i-ésimo nível de substituição (10, 20 e 30\%)) - (Nível do ingrediente (\%) na ração controle $(0 \%$ de substituição $) / 100 ; \mathrm{PFS}=$ preço por $\mathrm{kg}$ de farelo de soja $(\mathrm{R} \$ / \mathrm{kg}) ; \mathrm{PTB}=$ preço por $\mathrm{kg}$ de torta de babaçu $(\mathrm{R} \$ / \mathrm{kg}) ; \mathrm{POS}=$ preço por $\mathrm{kg}$ de óleo de soja $(\mathrm{R} \$ \mathrm{~kg})$; PDL-Met = preço por $\mathrm{kg}$ de DL-metionina $(\mathrm{R} \$ / \mathrm{kg})$; PL-Lis = preço por $\mathrm{kg}$ de L-lisina $\mathrm{HCl}(\mathrm{R} \$ / \mathrm{kg})$ e $\mathrm{PL}-\mathrm{Treo}=$ preço por $\mathrm{kg}$ de L-treonina $(\mathrm{R} \$ / \mathrm{kg})$.

As inequações foram utilizadas para simular a viabilidade da substituição do farelo de soja pela torta de babaçu considerando diferentes cenários de preços dos insumos.

\section{Resultados e Discussão}

As temperaturas média, máxima e mínima no interior das instalações durante o período experimental foram de $24,6 \pm 0,95 ; 28,2 \pm 1,56 \mathrm{e}$
$21,0 \pm 0,47{ }^{\circ} \mathrm{C}$, respectivamente, sendo a umidade relativa média do ar de $60 \%$. O que demonstrou que as aves permaneceram durante todo o período experimental dentro da zona de termoneutralidade, pois a temperatura e a umidade relativa do ar mantiveram-se dentro das condições ambientais consideradas confortáveis para esta categoria (MEDEIROS et al., 2005; OLIVEIRA et al., 2006).

Os níveis de substituição do farelo de soja pela torta de babaçu nas rações, não afetaram $(\mathrm{P}>0,05)$ o consumo de ração (CR), ganho de peso (GP), conversão alimentar (CA) e peso das aves aos 42 dias (P42d) (Tabela 3). Esses resultados estão relacionados ao fato das rações experimentais serem isoaminoacídicas, apesar do nível proteico ter variado entre os tratamentos, o que reforça a importância do conceito de "Proteína Ideal" em formulações para aves. Deste modo, é possível que as exigências nutricionais das aves tenham sido atendidas em todos os tratamentos avaliados, independentemente do nível de substituição do farelo de soja pela torta de babaçu.

Tabela 3. Valores médios de consumo de ração (CR), ganho de peso (GP), conversão alimentar (CA) e peso aos 42 (P42d) dias de frangos de corte, de acordo com o nível de substituição do farelo de soja pela torta de babaçu.

\begin{tabular}{lcccccc}
\hline \multirow{2}{*}{ Variáveis } & \multicolumn{2}{c}{ Níveis de substituição do farelo de soja pela torta de babaçu (\%) } & \multirow{2}{*}{$\mathrm{CV}^{1}$} & \multirow{2}{*}{$\mathrm{P}>\mathrm{F}^{2}$} \\
\cline { 2 - 4 } & 0 & 10 & 20 & 30 & & \\
\hline CR $(\mathrm{g})$ & 2789,45 & 2857,90 & 2815,06 & 2830,75 & 2,46 & 0,4897 \\
GP $(\mathrm{g})$ & 1458,25 & 1530,75 & 1415,94 & 1492,00 & 4,84 & 0,1427 \\
$\mathrm{CA}(\mathrm{g} / \mathrm{g})$ & 1,915 & 1,873 & 1,989 & 1,897 & 3,77 & 0,1507 \\
P42d (g) & 2347,28 & 2421,02 & 2305,63 & 2381,54 & 2,99 & 0,1330 \\
\hline
\end{tabular}

${ }^{1}$ Coeficiente de variação $(\%)$.

${ }^{2}$ Significância do Teste "F" da análise de variância.

Fonte: Elaboração dos autores.

Resultados semelhantes foram encontrados por Carneiro et al. (2009) quando avaliaram níveis crescentes de inclusão do farelo de babaçu (3, 6,9 e 12\%) em rações de frangos de corte e não verificaram efeitos sobre o consumo de ração, ganho de peso e conversão alimentar na fase de 21 a 42 dias de idade.
Observou-se que os níveis de substituição do farelo de soja pela torta de babaçu não afetaram $(\mathrm{P}>0,05)$ os pesos relativos da carcaça, coxa, sobrecoxa e peito dos frangos abatidos aos 42 dias (Tabela 4), indicando que a torta de babaçu pode ser incluída em até $30 \%$ em rações para frangos 
de corte, sem comprometer estas variáveis. Os resultados de rendimento de carcaça estão de acordo com os observados por Carneiro et al.
(2009), que não verificaram efeito dos níveis crescentes de farelo de babaçu sobre o rendimento de carcaça de frangos aos 42 dias de idade.

Tabela 4. Média dos rendimentos de carcaça (RC), coxa (RCX), sobrecoxa (RSCX) e peito (RP) de frangos de corte aos 42 dias de acordo com o nível se substituição do farelo de soja pela torta de babaçu.

\begin{tabular}{lcccccc}
\hline \multirow{2}{*}{ Variáveis } & \multicolumn{2}{c}{ Níveis de substituição do farelo de soja pela torta de babaçu (\%) } & \multirow{2}{*}{ CV $^{1}$} & \multirow{2}{*}{$\mathrm{P}>\mathrm{F}^{2}$} \\
\cline { 2 - 4 } & 0 & 10 & 20 & 30 & & 1,27 \\
\hline RC (\%) & 81,81 & 82,08 & 81,44 & 81,70 & 0,8118 \\
RCX (\%) & 12,22 & 11,82 & 11,91 & 12,41 & 4,65 & 0,3457 \\
RSCX (\%) & 14,12 & 13,72 & 13,18 & 14,16 & 5,23 & 0,1569 \\
RP (\%) & 33,45 & 34,29 & 33,53 & 33,45 & 3,63 & 0,6496 \\
\hline
\end{tabular}

${ }^{1}$ Coeficiente de variação (\%).

${ }^{2}$ Teste "F" da análise de variância.

Fonte: Elaboração dos autores.

Os níveis de substituição do farelo de soja pela torta de babaçu não afetaram $(\mathrm{P}>0,05)$ os pesos relativos do coração, moela e fígado, nem o peso relativo ou comprimento do intestino delgado (Tabela 5).

Tabela 5. Peso relativo das vísceras comestíveis (coração, moela e fígado) e intestino delgado e comprimento do intestino delgado $(\mathrm{m})$ de frangos de corte de 22 a 42 dias.

\begin{tabular}{|c|c|c|c|c|c|c|}
\hline \multirow{2}{*}{ Variáveis } & \multicolumn{4}{|c|}{ Níveis de substituição do farelo de soja pela torta de babaçu (\%) } & \multirow{2}{*}{$\mathrm{CV}^{1}$} & \multirow{2}{*}{$\mathrm{P}>\mathrm{F}^{2}$} \\
\hline & 0 & 10 & 20 & 30 & & \\
\hline Coração (\%) & 0,44 & 0,42 & 0,48 & 0,45 & 14,47 & 0,5576 \\
\hline Moela (\%) & 1,79 & 1,74 & 1,88 & 1,72 & 8,99 & 0,4181 \\
\hline Fígado $(\%)$ & 2,12 & 2,12 & 2,12 & 2,18 & 7,52 & 0,9204 \\
\hline Intestino delgado (\%) & 2,41 & 2,09 & 2,46 & 2,35 & 12,51 & 0,2529 \\
\hline Intestino Delgado (m) & 1,65 & 1,61 & 1,67 & 1,63 & 6,45 & 0,8347 \\
\hline
\end{tabular}

${ }^{1}$ Coeficiente de variação (\%).

${ }^{2}$ Significância do Teste " $F$ " da análise de variância.

Fonte: Elaboração dos autores.

Diversos autores (GONZÁLEZ-ALVARADO et al., 2007; GONZÁLEZ-ALVARADO et al., 2008; JIMÉNEZ-MORENO et al., 2009; JIMÉNEZ-MORENO et al., 2010; BRAZ et al., 2011; SACRANIE et al., 2012) verificaram que dietas com teores crescentes de fibra aumentaram a permanência do alimento no trato digestório das aves, aumentando o tamanho e o peso de diversos orgãos digestivos. Diante disso, esperava-se que os maiores teores de fibra, resultantes dos níveis crescentes de substituição do farelo de soja pela de torta de babaçu nas rações, pudessem influenciar o peso relativo dos órgãos das aves. Entretanto, esse comportamento não foi observado, indicando que os níveis de fibra nas dietas não atingiram magnitude suficiente para influenciar o desenvolvimento dos 
órgãos na fase final das aves.

Os resultados encontrados nesse trabalho indicaram que o aumento dos níveis de substituição do farelo de soja pela torta de babaçu nas rações, não prejudicou nenhuma das características de desempenho e rendimentos de carcaça, cortes ou órgãos dos frangos de corte de 22 a 42 dias de idade, evidenciando a viabilidade técnica da substituição de até 30\% farelo de soja pela torta de babaçu.

Em relação a análise de custo da alimentação, a maior margem bruta foi obtida com os frangos alimentados com $0 \%$ de torta de babaçu, em função do menor custo da alimentação (Tabela 6). O aumento dos níveis de substituição do farelo de soja pela torta de babaçu promoveu aumento no custo da alimentação, em decorrência do incremento de óleo de soja e aminoácidos sintéticos, visando manter o balanço energético e aminoacídico das dietas. Observa-se, com base nos preços dos insumos utilizados no presente experimento, que foi inviável substituir farelo de soja pela torta de babaçu na fase de 22 a 42 dias de idade.

Tabela 6. Ganho de peso (g), custo da alimentação ( $\mathrm{R} \$ / \mathrm{kg})$, custo da alimentação por kg de ganho de peso (R\$/kg), margem bruta $(\mathrm{R} \$ / \mathrm{kg})$ de frangos de corte de 22 a 42 dias.

\begin{tabular}{|c|c|c|c|c|}
\hline \multirow{2}{*}{ Variáveis } & \multicolumn{4}{|c|}{ Níveis de substituição do farelo de soja pela torta de babaçu (\%) } \\
\hline & 0 & 10 & 20 & 30 \\
\hline Ganho de peso (g) & 1458,25 & 1530,75 & 1415,94 & 1492,00 \\
\hline Custo da alimentação ${ }^{1}(\mathrm{R} \$ / \mathrm{kg})$ & 0,940 & 0,942 & 0,943 & 0,945 \\
\hline Custo da alimentação por $\mathrm{kg}$ de GP $(\mathrm{R} \$ / \mathrm{kg})$ & 1,801 & 1,804 & 1,807 & 1,810 \\
\hline Margem bruta $^{2}(\mathrm{R} \$ / \mathrm{kg})$ & 0,999 & 0,996 & 0,993 & 0,990 \\
\hline
\end{tabular}

${ }^{1}$ Considerando os seguintes preços: milho $=\mathrm{R} \$ 0,59 / \mathrm{kg}$; Farelo de soja $=\mathrm{R} \$ 1,30 / \mathrm{kg}$; Torta de babaçu $=\mathrm{R} \$ 0,70 / \mathrm{kg}$; Fosfato bicálcico $=\mathrm{R} \$ 2,77 / \mathrm{kg}$; Óleo de soja $=\mathrm{R} \$ 2,49 / \mathrm{kg}$; Calcário $=\mathrm{R} \$ 0,43 / \mathrm{kg} ;$ Sal = 0,80/kg; DL-metionina $=\mathrm{R} \$ 11,87 / \mathrm{kg} ; \mathrm{L}-\mathrm{lisina}$ $\mathrm{HCl}=\mathrm{R} \$ 6,67 / \mathrm{kg}$; L-treonina $=\mathrm{R} \$ 6,76 / \mathrm{kg}$; Suplemento mineral e vitamínico $=\mathrm{R} \$ 9,00 / \mathrm{kg}$.

${ }^{2}$ Considerando o preço do frango vivo pago em 08/03/2012 de R $2,80 / \mathrm{kg}$, pela empresa ASA Norte.

Fonte: Elaboração dos autores.

Entretanto, o custo da alimentação é variável, pois é influenciado pelas variações dos preços dos insumos impostos pelo mercado e pelas diferentes regiões do país. Diante disso, a substituição do farelo de soja pela torta de babaçu, pode ser viável economicamente em decorrências de modificações nos preços dos insumos das rações.

Considerando que a substituição de até $30 \%$ do farelo de soja pela torta de babaçu é tecnicamente viável, sua utilização em rações para frangos de corte dos 22 aos 42 dias de idade estará condicionada às seguintes desigualdades:

Se: $(0,0839 \times$ PFS $)>[(0,0839 \times$ PTB $)+(0,0093$ $\mathrm{x}$ POS $)+(0,0007 \mathrm{x}$ PDL-Met $)+(0,0024 \mathrm{x}$ PLLis $\mathrm{HCl})+(0,0016 \times$ PL-Treo $)]$ : substituir $30 \%$ do farelo de soja por torta de babaçu na ração é economicamente viável.

Se: $(0,0559 \times$ PFS $)>[(0,0559 \times$ PTB $)+(0,0062$ $\mathrm{x}$ POS $)+(0,0005 \mathrm{x}$ PDL-Met $)+(0,0016 \mathrm{x}$ PL-Lis $\mathrm{HCl})+(0,0007 \times$ PL-Treo $)]$ : substituir até 20\% do farelo de soja por torta de babaçu na ração é economicamente viável.

Se: $(0,0280 \times$ PFS $)>[(0,0280 \times$ PTB $)+(0,0031$ $\mathrm{x}$ POS $)+(0,0002 \mathrm{x}$ PDL-Met $)+(0,0008 \mathrm{x}$ PL-Lis $\mathrm{HCl})+(0,0004 \times$ PL-Treo $)]$ : substituir até $10 \%$ do farelo de soja por torta de babaçu na ração é economicamente viável.

Se: $(0,0280 \times$ PFS $) \leq[(0,0280 \times$ PTB $)+(0,0031$ $\mathrm{x}$ POS $)+(0,0002 \times$ PDL-Met $)+(0,0008 \times$ PL-Lis 
$\mathrm{HCl})+(0,0004 \times$ PL-Treo $)]:$ substituir farelo de soja por torta de babaçu na ração é economicamente inviável.

Sendo PFS = preço por kg de farelo de soja $(\mathrm{R} \$ /$ $\mathrm{kg}) ; \mathrm{PTB}=$ preço por $\mathrm{kg}$ de torta de babaçu $(\mathrm{R} \$ /$ $\mathrm{kg}) ; \mathrm{POS}=$ preço por $\mathrm{kg}$ de óleo de soja $(\mathrm{R} \$ / \mathrm{kg})$; PDL-Met = preço por $\mathrm{kg}$ de DL-metionina $(\mathrm{R} \$ / \mathrm{kg})$; $\mathrm{PL}-\mathrm{Lis} \mathrm{HCl}=$ preço por $\mathrm{kg}$ de L-lisina $\mathrm{HCl}(\mathrm{R} \$ / \mathrm{kg})$; $\mathrm{PL}-\mathrm{Treo}=$ preço por $\mathrm{kg}$ de L-treonina $(\mathrm{R} \$ / \mathrm{kg})$.

Se [1] for verdadeiro, a substituição de $30 \%$ do farelo de soja por torta de babaçu é economicamente viável. Caso [1] seja falsa, utiliza-se [2]. Se [2] for verdadeira, a substituição de até $20 \%$ do farelo de soja por torta de babaçu é economicamente viável. Se [2] for falsa, utiliza-se [3]. Se [3] for verdadeira, a substituição de até $10 \%$ do farelo de soja por torta de babaçu é economicamente viável. Se [3] for falsa, [4] será confirmada e a substituição do farelo de soja por torta de babaçu será economicamente inviável.

Utilizando essas inequações, foram feitas simulações da viabilidade de substituição do farelo de soja pela torta de babaçu para diferentes cenários de mercado:

Simulação 1: considerando os preços pagos pelos insumos das rações do presente estudo: PFS $=\mathrm{R} \$ 1,30(\mathrm{R} \$ / \mathrm{kg}) ; \mathrm{PTB}=\mathrm{R} \$ 0,70(\mathrm{R} \$ / \mathrm{kg}) ; \mathrm{POS}$ $=\mathrm{R} \$ 2,49(\mathrm{R} \$ / \mathrm{kg}) ; \mathrm{PDL}-\mathrm{Met}=\mathrm{R} \$ 11,87(\mathrm{R} \$ / \mathrm{kg})$; $\mathrm{PL}-\mathrm{Lis} \mathrm{HCl}=\mathrm{R} \$ 6,67(\mathrm{R} \$ / \mathrm{kg})$ e PL-Treo $=\mathrm{R} \$ 6,76$ ( $\mathrm{R} \$ / \mathrm{kg})$.

Para essas condições, verificou-se que, para que [1] seja verdadeira, e a substituição de 30\% do farelo de soja (FS) pela torta de babaçu (TB) seja viável economicamente, o maior valor que a TB pode assumir é R\$ 0,60 com os demais preços dos insumos constantes $(46,2 \%$ do valor do FS), como segue:

$(0,0839 \times 1,30)>[(0,0839 \times \underline{0,60})+(0,0093$ $\mathrm{x} 2,49)+(0,0007 \times 11,87)+(0,0024 \times 6,67)+$ $(0,0016 \times 6,76)] \rightarrow 0,10907>0,10863$; portanto [1] é Verdadeira e a substituição de $30 \%$ do FS pela TB será economicamente viável.

Caso a TB atinja preços superiores a $\mathrm{R} \$ 0,60$; [1] passa a ser falsa e [2] verdadeira até que a TB assuma um valor igual a R $0,64(49,2 \%$ do valor do FS $):(0,0839 \times 1,30)>[(0,0839 \times \underline{0,64})+(0,0093$ $\mathrm{x} 2,49)+(0,0007 \times 11,87)+(0,0024 \times 6,67)+$ $(0,0016$ x 6,76) $\rightarrow 0,10907<0,111986$; portanto [1] será Falsa e a substituição de $30 \%$ do FS pela TB será economicamente inviável; Utilizando [2]: $(0,0559 \times 1,30)>[(0,0559 \times \underline{0,64})+(0,0062 \times 2,49)$ $+(0,0005 \times 11,87)+(0,0016 \times 6,67)+(0,0007 \times$ $6,76)] \rightarrow 0,07267>0,072553$; portanto [2] será verdadeira e a substituição de $20 \%$ do FS pela TB será economicamente viável;

Caso a TB atinja o preço de R\$ 0,65 (50,0\% do valor do FS), [1] permanecerá falsa, [2] passará a ser falsa e [3] verdadeira, como segue:

$$
(0,0839 \times 1,30)>[(0,0839 \times \underline{0,65})+(0,0093 \times
$$
$2,49)+(0,0007 \times 11,87)+(0,0024 \times 6,67)+(0,0016$ $\mathrm{x} 6,76)] \rightarrow 0,10907<0,112825$;

Portanto [1] será Falsa; Utilizando [2]: $(0,0559 \mathrm{x}$ $1,30)>[(0,0559 \times \underline{0,65})+(0,0062 \times 2,49)+(0,0005$ $\mathrm{x} 11,87)+(0,0016 \times 6,67)+(0,0007 \times 6,76)] \rightarrow$ $0,07267<0,073112$;

Portanto [2] será Falsa; Utilizando [3]: $(0,0280 \mathrm{x}$ $1,30)>[(0,0280 \times \underline{0,65})+(0,0031 \times 2,49)+(0,0002$ $\mathrm{x} 11,87)+(0,0008 \times 6,67)+(0,0004 \times 6,76)] \rightarrow$ $0,0364>0,0363$;

Portanto [3] será Verdadeira e a substituição de 10\% do FS pela TB será economicamente viável.

Se a TB atingir um preço igual ou superior a R\$ 0,66 (50,8\% do valor do FS), as desigualdades [1], [2] e [3] não serão verificadas, confirmando [4], de modo que a substituição do farelo de soja pela torta de babaçu será economicamente inviável: $(0,0839 \mathrm{x}$ $1,30)>[(0,0839 \times \underline{0,66})+(0,0093 \times 2,49)+(0,0007$ $\mathrm{x} 11,87)+(0,0024 \times 6,67)+(0,0016 \times 6,76)] \rightarrow$ $0,10907<0,113664$; portanto [1] será Falsa; Utilizando [2]: $(0,0559 \times 1,30)>[(0,0559 \times \underline{0,66})$ $+(0,0062 \times 2,49)+(0,0005 \times 11,87)+(0,0016 \times$ $6,67)+(0,0007 \times 6,76)] \rightarrow 0,07267<0,073671$; 
portanto [2] será Falsa; Utilizando [3]: $(0,0280 \mathrm{x}$ $1,30)>[(0,0280 \times \underline{0,66})+(0,0031 \times 2,49)+(0,0002$ $\mathrm{x} 11,87)+(0,0008 \times 6,67)+(0,0004 \times 6,76)]$ $\rightarrow 0,0364<0,036613$; portanto [3] será Falsa; Utilizando [4]: $(0,0280 \times 1,30) \leq[(0,0280 \times \underline{0,66})+$ $(0,0031 \times 2,49)+(0,0002 \times 11,87)+(0,0008 \times 6,67)$ $+(0,0004 \times 6,76)] \rightarrow 0,0364<0,036613$; portanto [4] será verdadeira e a substituição do FS pela TB na ração será economicamente inviável, confirmando os resultados apresentados na Tabela 6 .

Simulação 2: considerando um aumento de 10\% no preço do farelo de soja: PFS = R \$ 1,43 $(\mathrm{R} \$ / \mathrm{kg})$; $\mathrm{POS}=\mathrm{R} \$ 2,49(\mathrm{R} \$ / \mathrm{kg}) ; \mathrm{PDL}-\mathrm{Met}=\mathrm{R} \$ 11,87(\mathrm{R} \$ /$ $\mathrm{kg})$; PL-Lis $\mathrm{HCl}=\mathrm{R} \$ 6,67(\mathrm{R} \$ / \mathrm{kg})$ e PL-Treo $=\mathrm{R} \$$ $6,76(\mathrm{R} \$ / \mathrm{kg})$.

Para essas condições, verificou-se: que se TB custar até R\$ 0,73 (51,0\% do valor do FS), [1] será Verdadeira e a substituição de $30 \%$ do FS pela TB será economicamente viável. Se TB custar entre R\$ 0,74 e R\$ 0,77 (51,7 a 53,8\% do FS); [1] será Falsa e [2] será Verdadeira, de modo que a substituição de $20 \%$ do FS pela TB será economicamente viável. Caso o preço da TB aumente para R $\$ 0,78$ (54,5\% do valor do FS), [1] e [2] serão Falsas e [3] será verdadeira, sendo economicamente viável a substituição de $10 \%$ do FS pela TB. Se o preço da TB for igual ou superior a R\$ $0,79(55,2 \%$ do valor do FS); [1], [2] e [3] serão Falsas e [4] será Verdadeira e a substituição do FS pela TB será economicamente inviável.

Simulação 3: Considerando uma aumento de $20 \%$ no preço do farelo de soja, PFS $=\mathrm{R} \$ 1,56(\mathrm{R} \$ /$ $\mathrm{kg}) ; \mathrm{POS}=\mathrm{R} \$ 2,30(\mathrm{R} \$ / \mathrm{kg}) ; \mathrm{PDL}-\mathrm{Met}=\mathrm{R} \$ 11,87$ $(\mathrm{R} \$ / \mathrm{kg}) ; \mathrm{PL}-\mathrm{Lis} \mathrm{HCl}=\mathrm{R} \$ 6,67(\mathrm{R} \$ / \mathrm{kg})$ e PL-Treo $=\mathrm{R} \$ 6,76(\mathrm{R} \$ / \mathrm{kg})$.

Com base nos mesmos procedimentos anteriormente descritos obtêm-se que: Se TB custar até R\$ 0,86 (55,1\% do valor do FS), [1] será Verdadeira e a substituição de $30 \%$ do FS pela TB será economicamente viável. Se TB custar entre R\$ 0,87 e R\$ 0,90 (55,8 a 57,7\% do FS); [1] será Falsa e [2] será Verdadeira, de modo que a substituição de $20 \%$ do FS pela TB será economicamente viável. Caso o preço da TB aumente para $\mathrm{R} \$ 0,91$ (58,3\% do valor do FS), [1] e [2] serão Falsas e [3] será verdadeira, sendo economicamente viável a substituição de $10 \%$ do FS pela TB. Se o preço da TB for igual ou superior a R $\$ 0,92(59,0 \%$ do valor do FS); [1], [2] e [3] serão Falsas e [4] será Verdadeira e a substituição do FS pela TB será economicamente inviável.

As inequações elaboradas no presente estudo mostraram-se coerentes e capazes de simular os efeitos da variação no preço dos insumos sobre a viabilidade econômica da utilização da torta de babaçu em substituição ao farelo de soja, em rações de frangos de corte dos 22 aos 42 dias de idade. Considerando que os preços dos insumos utilizados na nutrição de frangos de corte variam entre as diferentes regiões do país ao longo do ano, a utilização das inequações elaboradas no presente estudo pode ser de grande utilidade prática para produtores e nutricionistas, pois elas permitem verificar a viabilidade econômica da substituição do farelo de soja pela torta de babaçu, de acordo com o preço dos insumos, independente da região do país ou da época do ano.

\section{Conclusões}

A substituição do farelo de soja pela torta de babaçu é tecnicamente viável em até $30 \%$ em rações de frangos de corte dos 22 aos 42 dias, desde que as exigências nutricionais das aves sejam atendidas.

A substituição do farelo de soja pela torta de babaçu mostrou-se economicamente inviável para as condições do presente estudo, porém, as inequações aqui elaboradas podem ser de grande utilidade para simular a viabilidade econômica da substituição do farelo de soja pela torta de babaçu em diferentes cenários econômicos. 


\section{Agradecimentos}

Ao Conselho Nacional de Desenvolvimento Científico e Tecnológico - CNPq e a Fundação de Amparo a Pesquisa e ao Desenvolvimento Científico e Tecnológico do Maranhão - FAPEMA, pela concessão de bolsas de estudo.

\section{Referências}

BASTOS, S. C.; FUENTES, M. F. F.; FREITAS, E. R.; ESPÍNDOLA, G. B.; BRAGA, C. V. P. Efeito da inclusão do farelo de coco em rações para frangos de corte. Revista Ciência Agronômica, Fortaleza, v. 38, n. 3, p. 297-303, 2007.

BRAZ, N. M. de; FREITAS, E. R.; BEZERRA, R. M.; CRUZ, C. E. B.; FARIA, N. N. P.; SILVA, N. M. da; SÁ, N. L.; XAVIER, R. P. S. Fibra na ração de crescimento e seus efeitos no desempenho de poedeiras nas fases de crescimento e postura. Revista Brasileira de Zootecnia, Viçosa, v. 40, n. 12, p. 2744-2753, 2011.

BRUNELLI, S. R.; PINHEIRO, J. W.; SILVA, C. A. da; FONSECA, N. A. N.; OLIVEIRA, D. D. de; CUNHA, G. E.; SOUZA, L. F. A.de. Inclusão de farelo de gérmen de milho desengordurado na alimentação de frangos de corte. Revista Brasileira Zootecnia, Viçosa, v. 35, n. 4, p. 1349-1358, 2006.

CARNEIRO,A.P. M.; PASCOAL, L.A. F.; WATANABE, P. H.; SANTOS, I. B.; LOPES, J. M.; ARRUDA, J. C. B. de. Farelo de babaçu em rações para frangos de corte na fase final: desempenho, rendimento de carcaça e avaliação econômica. Ciência Animal Brasileira, Goiânia, v. 10, n. 1, p. 40-47, 2009.

GONZÁLEZ-ALVARADO, J. M.; JIMÉNEZMORENO, E.; LAZÁRO, R.; MATEO, G. G. Effect of type of cereal, heat processing of the cereal, and inclusion of fiber in the diet on productive performance and digestive traits of broilers. Poultry Science, Champaign, v. 86, n. 8, p. 1705-1715, 2007.

GONZÁLEZ-ALVARADO, J. M.; JIMÉNEZMORENO, E.; VALENCIA, D. G.; LÁZARO, R.; MATEOS, G. G. Effects of fiber source and heat processing of the cereal on the development and $\mathrm{pH}$ of the gastrointestinal tract of broilers fed diets based on corn or rice. Poultry Science, Champaign, v. 87, n. 9, p. 1779-1795, 2008.

JIMÉNEZ-MORENO, E.; GONZÁLEZ-ALVARADO, J. M.; GONZÁLEZ-SERRANO, A.; LAZÁRO, R.; MATEOS, G. G. Effect of dietary fiber and fat on performance and digestive traits of broilers from one to twenty-one days of age. Poultry Science, Champaign, v. 8, n. 12, p. 2562-2574, 2009.

Effects of type and particle size of dietary fiber on growth performance and digestive traits of broilers from 1 to 21 days of age. Poultry Science, Champaign, v. 89, n. 10, p. 2197-2212, 2010.

MEDEIROS, C. M.; BAÊTA, F. C.; OLIVEIRA, R. F. M.; TINÔCO, I. F. F.; ALBINO, L. F. T.; CECON, P. R. Efeitos da temperatura, umidade relativa e velocidade do ar em frangos de corte. Engenharia na Agricultura, Viçosa, v. 13, n. 4, p. 277-286, 2005.

NASCIMENTO, G. A. J. do; COSTA, F. G. P.; AMARANTE JÚNIOR, V. S.; BARROS, L. R. Efeitos da substituição do milho pela raspa de mandioca na alimentação de frangos de corte, durante as fases de engorda e final. Ciência Agrotecnologia, Lavras, v. 29, n. 1, p. 200-207, 2005.

OLIVEIRA, R. F. M.; DONZELE, J. L.; ABREU, M. L. T.; FERREIRA, R. A.; VAZ, R. G. M. V.; CELLA, P. S. Efeitos da temperatura e da umidade relativa sobre o desempenho e o rendimento de cortes nobres de frangos de corte de 1 a 49 dias de idade. Revista Brasileira de Zootecnia, Viçosa, v. 35, n. 3, p. 797-803, 2006.

PEREIRA, J. C.; SILVA, P. R. C.; CECON, P. R.; RESENDE FILHO, M. A.; OLIVEIRA, R. L. Cama de frango e suplemento à base de microbiota ruminal em dietas de novilhas leiteiras: desempenho produtivo e avaliação econômica. Revista Brasileira de Zootecnia, Viçosa, v. 32, n. 3, p. 653-662, 2003.

RAMOS, L. S. N.; LOPES, J. B.; FIGUEIRÊDO, A. V.; FREITAS, A. C.; FARIAS, L. A.; SANTOS, L. S.; SILVA, H. O. Polpa de caju em rações para frangos de corte na fase final: desempenho e características de carcaça. Revista Brasileira de Zootecnia, Viçosa, v. 35, n. 3, p. 804-810, 2006.

ROSTAGNO, H. S.; ALBINO, L. F. T.; DONZELE, J. L.; GOMES, P. C.; OLIVEIRA, R. F. de; LOPES, D. C.; FERREIRA, A. S.; BARRETO, S. L. de T. Tabelas brasileiras para aves e suínos composição de alimentos $e$ exigências nutricionais, Viçosa: UFV, Imprensa Universitária, 2011. $252 \mathrm{p}$.

SACRANIE, A.; SVIHUS, B.; DENSTADLI, V.; MOEN, B.; IJI, P. A.; CHOCT, M. The effect of insoluble fiber and intermittent feeding on gizzard development, gut motility, and performance of broiler chickens. Poultry Science, Champaign, v. 91, n. 3, p. 693-700, 2012.

SANTOS NETA, E. R. Avaliação de subprodutos do babaçu (Palmae orbignya martiana) na alimentação de frangos de corte. 2010. Dissertação (Mestrado em 
Ciência Animal Tropical) - Universidade Federal do Tocantins, Araguaína.

SANTOS NETA, E. R. dos; VAZ, R. G. M. V.; STATISTICAL ANALYSES SYSTEM - SAS. SAS/ RODRIGUES, K. F.; SOUSA, J. P. L.; PARENTE, I. INSIGHT User's guide. Versão 9.0 - versão para P.; ALBINO, L. F. T.; SIQUEIRA, J. C. de; ROSA, F. Windows. Cary: SAS Institute, 2002. CD-ROM. frangos de corte na fase inicial. Revista Brasileira Saúde Produção Animal, Salvador, v. 12, n. 1, p. 234-243, 2011. 